\title{
From Universal Laws of Cognition to Specific Cognitive Models
}

\author{
Nick Chater \\ Department of Psychology \\ University College London \\ Gordon D.A. Brown \\ Department of Psychology \\ University of Warwick
}

Please address correspondence to Nick Chater, Department of Psychology, UCL, Gower Street, London, WC1E6BT, n.chater@ucl.ac.uk.

Acknowledgements. Nick Chater and Gordon Brown were supported by research grant F/215/AY from the Leverhulme Trust. Nick Chater was also supported by a Leverhulme Senior Research Fellowship, and Gordon Brown by ESRC Grant RES 000 231038. We would like to thank Morten Christiansen, Jacob Feldman, Ulrike Hahn, Ian Neath, Roger Shepard, Paul Smolensky, Joshua Tenenbaum and Paul Vitányi. 


\begin{abstract}
The remarkable successes of the physical sciences have been built on highly general quantitative laws, which serve as the basis for understanding an enormous variety of specific physical systems. How far is it possible to construct universal principles in the cognitive sciences, in terms of which specific aspects of perception, memory, or decision making might be modelled? Following Shepard (e.g., 1987), it is argued that some universal principles may be attainable in cognitive science. Here we propose two examples: The simplicity principle (which states that the cognitive system prefers patterns that provide simpler explanations of available data); and the scale-invariance principle, which states that many cognitive phenomena are independent of the scale of relevant underlying physical variables, such as time, space, luminance, or sound pressure. We illustrate how principles may be combined to explain specific cognitive processes by using these principles to derive SIMPLE, a formal model of memory for serial order (Brown, Neath \& Chater, in press), and briefly mention some extensions to models of identification and categorization. We also consider the scope and limitations of universal laws in cognitive science.
\end{abstract}

Keywords: scale; scale-invariance; memory; free recall; serial recall; Kolmogorov complexity; similarity; generalization; universal laws; categorization 
A central question in cognitive science is the extent to which mental phenomena are subject to law-like regularities such as those observed in many aspects of the physical world. Additionally, how far might such regularities be general, or indeed, universal, in the same sense as, for example, the Newtonian principles that govern the movements of the planets, the behavior of falling bodies, and the properties of pendulums? The search for such laws has a long history. The German psychophysicists, for example, explored whether there were law-like dependencies in the process of sensory transduction (most notably leading to Weber's Law; and, more controversially, Fechner's Law). In the domain of learning, the programme of behaviorism took a strong universalist stance, aiming to formulate laws of learning (albeit typically in qualitative rather than quantitative form) concerning relationships between stimuli and responses that were intended to underpin the acquisition of all forms of behavior, in any species. While the scope of this program now appears to have been radically overambitious, it is nonetheless still true that theories of associative learning (e.g., Shanks, 1995) and their rivals (e.g., Gallistel \& Gibbon, 2000) provide quantitative and, potentially, general accounts of many learning phenomena.

The cognitive revolution, however, can be viewed as largely focussing attention away from the attempt to build universal laws. By viewing the mind as a highly complex computational device, it becomes natural to think of cognitive science as a process of 'reverse engineering'---or more specifically, 'reverse computer science'---rather than following in the mould of physics. Computer science does not seem to be full of quantitative universal laws---instead, its focus is on representations, and algorithms operating over those representations. Cognitive science has taken the same tack, exploring the multiple mental representations appropriate for perception, motor control, language, and common-sense reasoning, and considering computational frameworks, and specific processing algorithms, in which calculations over these representations can be defined. Thus, the projects of discovering how people recognize speech (e.g., Norris, McQueen \& Cutler, 2000), compute depth from stereo (e.g., Marr \& Poggio, 1976), or control the motor system (e.g., Wolpert \& Ghahramani, 2004). It is tempting to suspect that each such problem is sui generis; and that the mechanisms involved will have no more in common that the properties of the liver and the properties of the heart (e.g., 
Fodor, J. A., 1983). From this perspective, the quest for universal laws might seem inappropriate in the context of cognitive science, and as resulting from a false comparison with the physical sciences.

The correct locus for general principles in cognitive science, if any, might naturally be viewed as the computational architecture. Attempts to provide architectural frameworks which might provide a universal, or near-universal, structure into which specific cognitive theories may be implemented, have been central to the development of the field (e.g., production system architectures, e.g., Newell, 1990; Anderson, 1983; and various kinds of connectionist architectures, e.g., Rumelhart \& McClelland, 1986; as well as symbolic-connectionist hybrids of various kinds, e.g., Smolensky \& Legendre, 2006). Roger Shepard (Shepard, 1957, 1958a, 1958b, 1962a, 1962b, 1964, 1965, 1966, 1980, 1982, 1987, 1994), in a remarkable sequence of publications, has argued that, nonetheless, quantitative universal principles are possible in cognitive science---and indeed that such principles may serve as crucial building blocks for the construction of cognitive theories in specific domains. Two proposals have been particularly influential: Shepard's Universal Law of Generalization; and the "geodesic" account of mental transformations (e.g, Carlton \& Shepard, 1990a, 1990b). These proposals seek to drive quantitative laws into the very heart of central cognitive phenomena---which might seem to be governed by endlessly capricious representations and algorithms. In this paper, we will focus on the Universal Law, which will be central to the later discussion in this paper.

\section{Candidate Principles 1: Scale Invariance}

One of Shepard's most distinctive contributions to psychological theory has been an emphasis on the importance of symmetries in cognitive processes (e.g., Carlton \& Shepard, 1990a, 1990b; Farrell \& Shepard, 1981; Shepard \& Cooper, 1982; Shepard \& Zare, 1983). One of the most basic symmetries, and one that plays a central in the physical sciences, concerns scale. Scaling laws reveal so-called self-similarities: patterns that repeat in time, space, or other dimensions. From planetary motion, to shock waves and fluid flow, self-similarity is a ubiquitous feature of the physical world. Such patterns of scaling are so familiar that they may escape our notice. For example, as a pendulum is 
lengthened by a factor $f$, its dynamics are invariant, except that its period increases by a factor $\sqrt{f}$. Suppose that we record the pendulum's behavior; but we forget to record either spatial or temporal scale. Self-similarity implies that we can never recover this scale information. If our data are consistent with one combination of spatial and temporal scales, then they will be consistent with a combination in which the spatial scale is $f$ times larger; and time is flowing $\sqrt{f}$ times more slowly. Thus, the motion of the pendulum is scale-invariant. Remarkably, it turns out that many subtle problems in physics can be solved by making scale-invariance assumptions alone (Barenblatt, 1996) ${ }^{\mathrm{i}}$.

A particularly notable feature of self-similar phenomena is that the relevant descriptive laws are power laws, i.e., laws of the form:

$$
y=a x_{1}^{n_{1}} \ldots x_{i}^{n_{i}} \ldots x_{m}^{n_{m}}
$$

where $a$, and $n_{1}, \ldots n_{i}, \ldots n_{m}$ are arbitrary constants. Power laws embody self-similarity--their structure is the same, whatever scale we consider. That is, merely by looking at the form of the data, it is impossible to discover the absolute scale (and hence the units of measurement) of the variables $y$, and $x_{1}, \ldots x_{i}, \ldots x_{m}$. Figure 1 illustrates this point with a simple example $y=1 / x^{3}$. Note that the shape of the function is identical when we 'zoomin' on it, in this case by a factor of two on the $x$-axis, and by a factor $2^{3}=8$ on the $y$-axis. Note that any other functional relationships between variables are not scale invariant. For example, exponential decay has a specific scale (the 'half-life'); and the Gaussian has a specific scale (i.e., standard deviation). While scale-invariance is well-enough understood to be widely practically applied throughout the physical sciences; nonetheless, its precise conceptual foundationa are still a question for active research (e.g., Barenblatt, 1996).

Physics aside, scale-invariance applies across a wide range of social/economic (e.g., Gabaix, 1999; Ijiri \& Simon, 1977; Mantegna \& Stanley, 1995), biological (e.g., Gisiger, 2001; Goldberger, Amaral, Hausdorff, Ivanov, Peng \& Stanley, 2002; West \& Brown, 2005) and cognitive phenomena. In cognitive science, scale invariance applies to many of the best claims to be psychological laws (Table 1). Thus, the power laws of forgetting, the power law of practice, and Stevens' law (that perceptual inputs and 
judgments are related by a power law) are instances of scale invariance. So too is Weber's Law, which states that the precision of the encoding of a stimulus is proportion to the magnitude of a stimulus. Weber's Law is an example of scale-invariance, because the ratio of precision and absolute magnitude is constant---and hence this ratio is the same at all scales (Figure 2). Weber's Law is, of course, violated for extremely stimulus values, where the sensory system is either overloaded, or at the limits of stimulus detection. In both cases, it is the departures from scale-invariance---cognitive bumps--that are revealing about underlying cognitive mechanisms.

There is also a wide range of phenomena which have a complex pattern that is invariant over changes of scale. For example, patterns of recall often appear invariant to changes in time-scale, in a range of paradigms. Maylor, Chater and Brown (2001) asked people to recall events that had happened in the last day, week or year, and found that the speed at which they were able to retrieve these memories was the same for each condition. Of course, the type of event recalled at each time-scale was very different (e.g., we might recall a holiday, in the 'year' condition; or eating breakfast in the 'day' condition). Had people not made this 'significance' adjustments, participants could clearly have retrieved more items for the longer time periods, as any event recalled in the last day is a fortiori a memory from the last week and year. In fact, people adjusted what counts as significant in a way that maintained scale-invariance---the rate of retrieving items was independent of time-scale (Figure 3). As also shown in Figure 3, the same effect occurred with prospective memories, i.e., memories for things that will happen in the future.

Another example concerns the shape of serial position curves in serial and free recall which may exhibit scale invariance with respect to time. Scale-invariance is consistent, in particular, with the much discussed ratio rule: that the slope of the recency curve is determined by the ratio of the rate at which items are presented and the time since the last item (e.g., Bjork \& Whitten, 1974; Glenberg et al. 1983; but see Davelaar, Goshen-Gottstein, Ashkenazi, Haarmann, \& Usher, 2005). Thus, if the entire schedule of learning and test is scaled multiplicatively (see Figure 4), then the slope of the recency curve should be unchanged. Similar effects are seen in memory for temporal order. Neath and Brown (2006) note that when the position of items separated by $50 \mathrm{~ms}$ in a list must 
be recalled, or the day of the week on which an event occurred must be remembered, similar serial position curves are observed over timescales that vary by six orders of magnitude.

Finally, note that scale-invariance is ubiquitous in many aspects of the perceptual and motor system. In the context of perception, scale invariance is sometimes so natural that it is scarcely noticed---for example, object, text, or face recognition is roughly invariant to the retinal size of the stimulus; recognition of melodies is roughly invariant both to the volume and pitch, and so on. These invariance of scale are, of course, part of a wider set of symmetries (e.g., in the visual world, involving translations and rotations), which Shepard and colleagues have explored, both in perception and imagery (e.g., Farrell \& Shepard, 1981; Shepard \& Cooper, 1982). Similarly, scale invariance is widespread in the motor system. For example, Schmidt, Zelaznik, Hawkins, Frank and Quinn (1979) developed a motor analog of Weber's Law, showing that the variable of a forced produced scales linearly with the magnitude of that force; and both simple movements (e.g., pointing, Soechting \& Lacquaniti, 1981) and extremely complex movements (e.g., handwriting, Viviani \& Terzuolo, 1980) are scale-invariant in both space and time.

We have argued that scale-invariance applies across a wide range of cognitive phenomena. Indeed, we suggest that, unless there is reason to suppose that some aspect of the environment and agent depends on a specific scale (as, for example, in speech processing) scale-invariance may be expected. Thus, a wide variety of psychological regularities, many of which are viewed as requiring special theoretical explanation, may be viewed as coming from a common source, the symmetry induced by the lack of any special phenomena at any particular scale. We suggest, therefore, that it is departure from, rather than adherence to, scale-invariance, that requires theoretical explanation.

Thus, for example, Gilden, Thornton and Mallon (1995) asked people to tap each time they thought that a specific time interval had elapsed (in the range 0.3 to $10 \mathrm{~s}$ ). They then calculated the temporal displacement from a "regular" rhythm from each successive interval. These displacements showed correlations across a wide range of time-scales--and indeed, the power spectrum of revealed a power law, which is characteristic of scaleinvariance (the exponent of this power law was roughly -1, a noise-structure often 
observed in physical data, Handel \& Chung, 1993). Crucially, though, the power spectrum also shows a well-defined kind, at high temporal frequency, after which the power spectrum has the flat structure characteristic of white noise. Gilden et al. (1995) argued that this kink, indicating a violation of scale invariance, is evidence for two cognitive processes underlying performance: a scale-invariance timing mechanism combined with high frequency "jitter" from the motor system, a viewpoint for which there is independent support (e.g., Wing, 1980).

Violations of scale-invariance may be informative at a qualitative level, too. Whereas the human auditory system generally processes acoustic signals in a similar way across a wide range of temporal frequencies (i.e., reflecting scale-invariance), there may be characteristic psychophysical transitions around frequencies where the dominant coding mechanism for frequency changes (i.e., around 4-5 kHz, after which at which neural phase locking does not occur, Moore, 2003). And whereas music and ambient sounds typically sound qualitatively similar when shifted in frequency, speed sounds that are shifted in frequency typically sound utterly bizarre. This presumably arises because there is a characteristic band of frequencies in human speech, which is itself a function of the fact that the human vocal chambers have a characteristic size, rather than being scaleinvariant (see Lewicki, 2002). The fact that scale-invariance in frequency is violated for human speech processing is, therefore, interesting and informative; the maintenance of scale-invariance for many other types of stimuli is, by contrast, merely the natural default. More broadly, we suggest that violations of scale-invariance are likely to be especially revealing of the underlying neural and cognitive mechanisms.

\section{Candidate Law 2: The Simplicity Principle}

Many problems faced by the cognitive system can be viewed as a type of inductive inference---as finding patterns in data. The perceptual system finds patterns, providing information about the external world, from input to the sensory receptors. Acquiring a language involves finding the wide variety of levels of structure described by linguistic theory. Learning to classify items, on the basis of experience, involves deriving category structures from experience (Feldman, 2000; Tenenbaum, 1999). 
Inductive inference problems of this form can be formulated in terms of Bayesian inference (e.g., Chater, Tenenbaum \& Yuille, 2006). Accordingly, the probability of each pattern or structure, after the data is received, is proportional to the product of the conditional probability of the data, given that structure (i.e., how well the structure 'fits' the data); and the prior probability of that structure. A central issue in the Bayesian program is how these prior probabilities should be set.

One approach is to set the priors using simplicity---specifically, we assume that the probability of a model, grammar, or pattern is inversely proportion to its complexity. How can this intuition be made precise? The mathematical theory of Kolmogorov complexity (Li \& Vitányi, 1997) provides a natural framework. The complexity of a formal object, $x$, is defined as the length of the shortest code in a universal programming language that generates $x$. This code length, the Kolmogorov complexity of $x$, is written $K(x)$. A variety of mathematical considerations ${ }^{\mathrm{ii}}$ suggest that the most natural prior distribution over possible probabilistic models, $M$, of the data is proportional to $2^{-K(M)}$ (deterministic models are, of course, merely a special case in which all probabilities are 0 or 1). ${ }^{\text {iii }}$ This conclusion can be viewed as a mathematical version of Occam's razor---that, other things being equal, simple theories should be preferred.

Suppose we collect data $D$. From a probabilistic standpoint, a natural objective is to choose the most probable model, i.e., the $M$ that maximizes $\operatorname{Pr}(M \mid D)$. By Bayes' theorem, we know that this will be the $M$ that maximizes:

$$
\operatorname{Pr}(D \mid M) \operatorname{Pr}(M)
$$

which, substituting the universal prior into (2), gives:

$$
\operatorname{Pr}(D \mid M) 2^{-K(M)}
$$

and, of course, the $M$ that maximizes (3), also maximizes

$$
\log _{2}\left(\operatorname{Pr}(D \mid M) 2^{-K(M)}\right)
$$


by the monotonicity of $\log$. This $M$ therefore also minimizes

$$
\begin{aligned}
& -\log _{2}\left(\operatorname{Pr}(D \mid M) 2^{-K(M)}\right) \\
& =-\log _{2}(\operatorname{Pr}(D \mid M))+K(M)
\end{aligned}
$$

An elegant result from Kolmogorov complexity theory is that, for a computable probability distribution $\operatorname{Pr},-\log _{2} \operatorname{Pr}(x)=K(x)$ up to a constant term independent of $x$, for almost all $x$ (strictly, with probability of measure 1, if the $x$ are drawn from Pr---i.e., there are counterexamples, but they are rare). ${ }^{\text {iv }}$ An analogous result holds for conditional probabilities: i.e., with high probability:

$$
-\log _{2} \operatorname{Pr}(D \mid M)=K(D \mid M)
$$

where the conditional Kolmogorov complexity, $K(y \mid x)$, is the length of the shortest program that yields $y$, given $x$ as input. Roughly, conditional complexity is small where there is a simple transformation which maps input to output---we shall see that this notion of simple transformation provides the basis for an interesting general notion of similarity, below, in our discussion of the universal law of generalization.

Combining (5) and (6), we conclude that, with high probability, the models $M$ with maximal, or near maximal, posterior probability, will also be the $M$ with minimal code length, i.e., in symbols:

$$
\underset{M_{1}}{\arg \max } \operatorname{Pr}\left(M_{1} \mid D\right)=\underset{M_{2}}{\arg \min } K\left(M_{2}\right)+K\left(D \mid M_{2}\right)
$$

This derivation, which is derived and presented rigorously by Vitányi and Li (2000), can be informally stated as follows: Models that have high a priori probability according to a Bayesian analysis using any computable prior probability, will (with high probability) correspond to models which allow short two-part descriptions of the data, and vice versa. This result ${ }^{\mathrm{v}}$ provides a fundamental normative underpinning for the simplicity principle-- 
-that the cognitive system should prefer models that provide the shortest codes for the data, where such codes consist of (i) a specification of the model or hypothesis; and (ii) an encoding of the data, given that model.

In the light of the close relationship between Bayesian and simplicity-based reasoning, how should we conceive of the relationship between them? Some theorists, both in statistics (Rissanen, 1987) and cognitive science (Leeuwenberg \& Boselie, 1988) argue that simplicity should be viewed as basic---essentially because the Bayesian approach attempts to assign probabilities of regularities in the "real" world, and it may be doubted that such regularities are, at least outside fundamental physics, likely to be anything more than approximations. Others, in machine learning (e.g., Wallace \& Freeman, 1987) and cognitive science (Chater, 1996; Mumford, 1996) view simplicity and Bayesian inference as equivalent - and view the choice of theoretical framework as a matter of methodological convenience. A simplicity approach is particularly useful in situations where it is easier to form hypotheses about cognitive representations than to form hypotheses about appropriate probability distributions. For example, theories about the representation of formal languages provide a framework for coding grammars; and hence this framework will induce code-lengths for such grammars. But it is arguable less clear how directly to specify a probability distribution over grammars, and hence in this case, a simplicity-based perspective may be more useful. Conversely, specific background knowledge, or statistical information, is easier to incorporate in a probabilistic framework. Hence a Bayesian framework may be more appropriate for ecological stimuli (e.g., natural textures or images), where underlying physical regularities are fairly well-understood (e.g., concerning the physical regularities, including optics, that generate natural images, Richards, 1988) and where statistical properties of the stimuli can be measured empirically (e.g., Field, 1987). ${ }^{\mathrm{vi}}$

This result provides reason to believe that the simplicity principle is a reasonable principle of inductive inference. Another normative justification concerns prediction, which arises in the slightly different setting in which a corpus of data accumulates at each time step. A fundamental result, which we call the prediction theorem (Solomonoff, 1978), shows that for any sequence of data produced by a computational process (which can be combined with randomness), prediction of the next item based on simplicity 
converges with high probability on the 'true' probabilities. Specifically, the expected sum-squared error of the infinite number of predictions of the next item, as the sequence unfolds, is bounded by a finite sum, proportional to the Kolmogorov complexity of the data-generating process (see Li \& Vitányi, 1997) which can be viewed as indicating the possibility of induction, under very general conditions.

How far, though, is the simplicity principle not merely normatively justified but a useful description of how the cognitive system finds structure? A direct test is difficult because detailed predictions from the principle in any domain appears to require having independent, and fairly detailed, knowledge of the relevant mental representations--because code lengths are defined in terms of these representations. ${ }^{\text {vii }}$

Now, one of the attractions of the theoretical notion of Kolmogorov complexity is that it allows us to abstract away from details concerning the precise coding language (as long as the language is sufficiently powerful---i.e., as powerful as a universal programming language, which turns out to be a surprisingly low hurdle). Indeed, the celebrated invariance theorem (see Li \& Vitányi, 1997) shows that, for any two coding languages, the difference between their code lengths, for all objects that can be coded at all, cannot exceed a constant. This result allows us to abstract away from coding languages, and make mathematical progress (just as computational complexity theory, Garey \& Johnson, 1979), allows computer scientists to describe the time-complexity of algorithms, independent of the details of the machine on which the algorithms run). We shall see the usefulness of this level of abstraction, in considering Shepard's Universal Law, and psychological models to which it can be related, below.

From the point of view of providing detailed psychological predictions, however, the need for a theory of mental representation, in terms of which codes can be constructed, remains (just as, if we are interested in building a model of reaction times for a particular task, assumptions concerning the specific computational and neural machinery involved will be of crucial importance; computational complexity theory is not, of course, enough). Fortunately, however, a great deal of work within cognitive science has been devoted to building theories of mental representation, in particular domains; for example, in the case of language processing, linguistics provides a rich set of potential levels of description, which have been enriched, modified and extended by 
work in psycholinguistics and computational linguistics. Similarly, psychological, neuroscientific and computational, work has provided a rich range of hypotheses concerning the representation of the perceptual world. Given a representational system, the simplicity principle predicts that the cognitive system will prefer to encode the available data using the structure or model that provides the shortest encoding of the available data. This program has been investigated by a range of formal and computational models, in language (e.g., Brent \& Cartwright, 1996; Dowman, 2000; Goldsmith, 2001) and perception (e.g., Attneave \& Frost, 1969; Bienenstock, Geman \& Potter, 1998; Hochberg \& McAlister, 1953; Leeuwenberg, 1971).

Keeping the argument at a general level, however, still allows us to draw on number of lines of evidence that appear consonant with the simplicity viewpoint (Table 2) (that have been reviewed elsewhere, see Chater, 1999; Chater 2005; Chater \& Vitányi, 2003). For example, a vast range of phenomena in perceptual organization, including the Gestalt laws of closure, good continuation, common fate, and so on, have been widely interpreted as revealing a preference for simplicity (see Figure 5). Moreover, grouping effects that may be determined by simplicity also have wide ramifications for other aspects of perception (e.g., Adelson, 2000). Items with simple descriptions are typically easier to detect in noise and easier to detect (Garner, 1974). Finally, note that the physiology of early vision, including receptive field shapes, and phenomena such as lateral inhibition, seems adapted to maximize information compression in vision (Blakemore, 1990).

Moving to higher level cognition, the simplicity of a code for a stimulus is related to the amount of structure uncovered in that stimulus. The more structure people can find in a stimulus, the easier they find it to process and remember and the less random it appears (Falk \& Konold, 1997; see also Griffiths \& Tenenbaum, 2003). The speed of learning for Boolean concepts (e.g., A OR B OR C; A AND (B OR C) etc) is well predicted by the shortest code length for those concepts (Feldman, 2000, 2006). Moreover, as we shall now see below, the simplicity principle provides a natural machinery for building a general theory of similarity (Chater \& Vitányi, 2003; Hahn, Chater \& Richardson, 2003). 
The simplicity principle relates closely to a number of other approaches to inference. As we noted in deriving (7), the simplicity principle typically favours models with high Bayesian a posteriori probability. Indeed, the simplicity principle can be viewed as corresponding to Bayesian inference, using a particularly general "ignorance"

prior---i.e., one for which hypotheses, $H$, have a prior probability given by $2^{-K(H)}$, where $K(H)$ is the length of the shortest code for $H{ }^{\text {viii }}$ The simplicity principle, as stated in terms of Kolmogorov complexity, is a highly idealized notion; practical statistical methods based on simplicity "scale-down" the approach, to consider the shortest code length given restricted coding schemes, typically assuming, for example, that data is independently drawn of each trial from a single distribution; that it is generated from some particular class of probability distributions, etc. Statistical perspectives include Rissanen's (1987) Minimum Description Length, Wallace and colleagues’ Minimum Message Length (Wallace \& Freeman, 1987), and Dawid's (1984) prequential approach to statistical inference.

Given these close relationships between concepts based on coding and based on probability, a natural question is: which should be viewed as basic? We suggest that, from a theoretical point of view, coding and probabilistic concepts may be viewed as equivalent; but that, in practice, the most workable framework should be selected. In many areas of cognition, we suggest, we can make reasonable assumptions about mental representations (especially in language and in early vision), and, indeed, much of cognitive psychology has been oriented towards questions of representation. In such cases, viewing coding and simplicity as basic, and probability as a derived notion, may be most appropriate.

\section{The Universal Law of Generalization: A Derivation from Simplicity} The Universal Law of Generalization (Shepard, 1987) considers the question of generalization from observation of one item, $S_{i}$, to a second item, $S_{j}$. In abstract terms, this can be viewed as a problem of induction, with just one prior specific instance (Heit, 2000). That is, suppose that I learn that Daisy the cow has property $X$; how likely do I believe it is Bill the horse has property $X$; or that Herbie the car has property $X$. At first sight, it might seem that type of problem is entirely resistant to quantitative analysis (e.g., 
Bush \& Mosteller, 1951; Fodor, 1983; Oaksford \& Chater, 1998; Pylyshyn, 1987). Such problems appear to depend crucially on how different kinds of object are represented and the nature of the property $X$ under consideration. Furthermore, the process of inductive inference can potentially depend on arbitrary amounts of relevant background knowledge

The elegance of the Universal Law of Generalization is that it aims, not to deny, but to cut through such complexity. The proposal is that the probability of generalizing from one stimulus item, $S_{a}$, to another, $S_{b}$, is proportional to the negative exponential of the psychological distance, $D(a, b)$, between them. In symbols:

$$
G(a, b)=A e^{-B D\left(S_{a}, S_{b}\right)}
$$

The elegance of the approach is that, while both quantities may be influenced by any number of specific representational or algorithmic constraints, the law states that they will be influenced in corresponding ways. Specifically, if, for whatever complex reasons, two items are nearby in psychological space, then generalization between them will be high; if they are not, generalization will be low.

For the Universal Law of Generalization to be practically useful, it is of course crucial to be able to independently measure the psychological distance between pairs of items. A key breakthrough in achieving this arises from Shepard's pioneering work on non-metric multi-dimensional scaling. This method takes as input an ordering of the distances between all pairs of items. That is, all that is required is that we can determine whether the psychological distance between, say, $A$ and $B$, is greater or less than the distance between $C$ and $D$; no 'metric' assumptions are required concerning the size of these distances or the size of the difference between them. The output is a 'map' with each point embedded in a (typically Euclidean) space, where each item is located in the space so that the ordering of the distances between points matches the ordering of distances that were the original data. Thus, this space now provides an estimate of the relative psychological distance between the items; and this measure of psychological distance can be used to make predictions concerning generalization.

The key ideas behind the simplicity principle can also be applied to provide a general picture of similarity and confusion between them. Intuitively, it is natural to 
suggest that the similarity between two representations is, to some degree, related to the complexity of the transformation required to turn each representation into the other. Thus, two nearby points in a geometric space can be viewed as similar, because a small shift in locations suffices to map one to the other; and a pair of items with highly overlapping features may be viewed as similar, because relatively few 'flips' will be required to map one set of features onto the other. This general idea can, of course, apply to representations of any kind: We may view two sentences as similar if they can be related by a simple grammatical transformation; or two pictures of the same object may be viewed as similar if they can be related by a change of viewpoint or lighting.

These intuitions are the starting point for the Representational Distortion theory of similarity (Hahn, Chater \& Richardson, 2003), according to which the dis-similarity between a pair of mental representations $x$ and $y$ is a function of the complexity of the transformation between them, given a particular representational coding language. The conditional Kolmogorov complexity, $K(x \mid y)$, the length of the shortest code that transforms $y$ into $x$, is the natural starting point for this kind of account, although a variety of specific formulations are possible (e.g., imposing symmetry or allowing asymmetry; applying various types of normalization; factoring apart structure from noise for each object, and so on). Just as the invariance theorem, described above, allows us to abstract away from specific coding languages, and just speak of the complexity of a particular an object, $x$, i.e., $K(x)$, so the analogous result for conditional Kolmogorov complexity, allows to us abstract away from specific coding languages, and speak of the complexity of a transformation between two objects, $K(x \mid y)$. Note that we do not have to assume a specific coding languages for transformations - rather we can merely appeal to the representation language in terms of which the objects $x, y$ and so on are coded. As we noted above, while this abstraction is mathematically useful (as we shall see shortly), precise behavioral predictions concerning the similarities of individual stimuli will, of course, depend on the coding language used, just as we described above, when describing the simplicity principle. Of course, the same goes for feature-based (Tversky, 1977) or geometric theories of similarity (Shepard, 1980)—only when specific features, or dimensions, are specified for the set of objecs under consideration, can we make precise 
behavioral predictions, even though general properties of the accounts can be analysed in the absence of such detailed information.

Hahn et al. (2003) provide empirical evidence for the representational distortion account, in experiments in which people judge the similarities of three dimensional arrangements of blocks (items which, they argue, cannot readily be captured in either Euclidean or featural terms, and need a more general 'structured' representation). The account of similarity is attractive because it provides a general notion of similarity which may be relevant to wide variety of representations used in cognitive science (e.g., Fodor \& Pylyshyn, 1988; Russell \& Norvig, 2003; Tenenbaum, Griffiths \& Niyogi, 2007); and also because it collapses into geometric and feature-based models of similarity, in special cases.

Here, though, we focus instead on more formal aspects of this approach. Conditional Kolmogorov complexity can be the basis for a "universal" measure of (dis)similarity, $D_{U}$, the 'information metric' (Bennett, Gács, Li, Vitányi \& Zurek, 1998), which can be defined as: ${ }^{\text {ix }}$

$$
D_{U}(x, y)=1 / 2(K(y \mid x)+K(x \mid y))
$$

This metric is universal in the sense that, if any cognitively plausible distance measure treats two items as similar, the information metric also treats them as similar. Specifically, if we impose some mild restrictions on the notion of distance---call these admissible distances, ${ }^{\mathrm{x}}$ the informational distance is, in a sense, a minimal distance between any pair of items (for discussion, see Chater \& Vitányi, 2003; for technical details, see Bennett et al., 1998).

More formally, an admissible distance $D(x, y)$ is universal if, for every admissible distance $D^{\prime}(x, y)$, we have

$$
D(x, y) \leq D^{\prime}(x, y)+c_{D}
$$

where $c_{D}$ is a constant that depends on $D$, but not on $x$ and $y$. It turns out that, remarkably, that universal distances exist; and that $D_{U}(x, y)$ is, indeed, a universal distance. This 
implies that it assigns nearly as small a distance between two objects as any cognitive distance will do. Thus, for example, while the positive and negative image of the same picture are far away from each other in terms of Euclidean distance (because each pixel value is different), they are at almost zero distance in terms of universal distance because interchanging the black and white pixels transforms one picture into the other. The universal similarity metric is, like other notions rooted in Kolmogorov complexity, an 'ideal' notion in the sense that it ignores the limitations on processing capacity, or the goals of the cognitive system.

$D$ may therefore be viewed as a "default" distance metric---that is, if we know nothing about the particular similarity metric that the cognitive system uses, in some context, we know at least that it is not "too far from" $D$. Yet, as we noted above $D$ can also be viewed as providing a positive theory of similarity---a theory that states that cognitive distance is determined by the complexity of the transformations required to turn the representation of one object into the representation of the other, and vice versa. Rather than describe empirical evidence of this approach here (Hahn, Chater \& Richardson, 2003), we focus on how the application of simplicity to similarity can generate non-trivial psychological generalizations. Specifically, and especially relevant to the theme of this Special Issue, Shepard's contribution to cognitive science, we show that psychological distance between mental representations yields Shepard's Universal Law of Generalization (Chater \& Vitányi, 2003). ${ }^{\mathrm{xi}}$

Although intended to have broader application, the Universal Law of Generalization is primarily associated with a specific experimental paradigm - the identification paradigm (for other derivations of the Universal Law, focusing on generalization rather than confusability, see Shepard, 1987; Tenenbaum \& Griffiths, 2000). In this paradigm, humans or animals are repeatedly presented with stimuli concerning a (typically small) number of items. We denote items, e.g., phonemes, colours, or tones, as $a, b, x$, the representations of the corresponding perceptual stimuli as $S_{a}, S_{b}, S_{x}$; and the representation of the corresponding distinct responses as $R_{a}, R_{b}, R_{x}$. In the identification paradigm, experimental participants are required to associate a specific, and distinct, response with each item - a response that can be viewed as 'identifying' the item concerned. The stimulus $S_{a}$ is associated should evoke response $R_{a}$. In practice, this 
occurs only probabilistically - the more similar $a$ and $b$ are, the greater the chance that $S_{a}$ might evoke $R_{b}$. We leave open, for now, the question of whether these responses arise from confusion of perception, or memory, or through deliberate generalization from one item to another.

Shepard uses a specific, symmetric, measure, $G(a, b)$, to capture what he terms the 'generalization' between items a and $b$.

$$
G(a, b)=\left(\frac{\operatorname{Pr}\left(R_{a} \mid S_{b}\right) \operatorname{Pr}\left(R_{b} \mid S_{a}\right)}{\operatorname{Pr}\left(R_{a} \mid S_{a}\right) \operatorname{Pr}\left(R_{b} \mid S_{b}\right)}\right)^{1 / 2}
$$

Now we are in a position to directly relate $G(a, b)$ to information distance.

Recall that, for a computable probability distribution $\operatorname{Pr}$, with high probability, $-\log _{2} \operatorname{Pr}(x)$ $=K(x)$ up to a constant term independent of $x$.

$$
\log _{2} G(a, b)=1 / 2 \log g_{2} \operatorname{Pr}\left(R_{a} \mid S_{b}\right)+\log _{2} \operatorname{Pr}\left(R_{b} \mid S_{a}\right)-\log _{2} \operatorname{Pr}\left(R_{a} \mid S_{a}\right)-\log _{2} \operatorname{Pr}\left(R_{b} \mid S_{b}\right)
$$

We assume mapping an item onto itself can be achieved by a fixed finite program, independent of the particular items $a$ and $b$ (this is essentially an identity mapping) ) $^{\mathrm{xi}}$, and hence the terms $-\log _{2} \operatorname{Pr}\left(R_{a} \mid S_{a}\right)$ and $-\log _{2} \operatorname{Pr}\left(R_{b} \mid S_{b}\right)$ can be collapsed into the $o(1)$ term. Replacing the $\log _{2} \operatorname{Pr}\left(R_{a} \mid S_{b}\right)$ with $K\left(R_{a} \mid S_{b}\right)$, and $\log _{2} \operatorname{Pr}\left(R_{b} \mid S_{a}\right)$ with $K\left(R_{b} \mid S_{a}\right)$, we obtain:

$$
\log _{2} G(a, b)=-1 / 2 k\left(R_{a} \mid S_{b}\right)+K\left(R_{b} \mid S_{a}\right) \underset{z}{\mathbf{Z}} o(1)
$$

where the $o(1)$ indicates that (12) holds up to a constant. Assuming that there is a fixed program that maps the one-to-one correspondence between the $S_{x}$ to $R_{x}$, this means that complexities will be invariant (up to a constant, and depending on the complexity of this mapping) if responses are replaced with stimuli, throughout (or, indeed, vice versa). This 
is typically a reasonable assumption---e.g., in phoneme identification, the correspondence between the sound /b/ and the production of $\mathrm{a} / \mathrm{b} /$ sound by the learner can be taken as built in, before the experiment begins. In an identification paradigm, it is particularly natural, because we assume that the participant has learned the labels of the stimulus items during a training phase. In cases where the mapping is complex and must be learned, collapsing the $S_{x}$ to $R_{x}$ would be inappropriate. ${ }^{\text {xiii }}$ Making this substitution and applying (9), we obtain:

$$
\begin{aligned}
& \log _{2} G(a, b)=-1 / 2\left(S_{a} \mid S_{b}\right)+K\left(S_{b} \mid S_{a}\right) \frac{7}{7} o(1) \\
& =-D_{U}\left(S_{a}, S_{b}\right) \pm o(1)
\end{aligned}
$$

Rearrangement leads to:

$$
G(a, b)=A e^{-B D_{U}\left(S_{a}, S_{b}\right)}
$$

where $A$ and $B$ are arbitrary constants ${ }^{\text {xiv }}$. This is the Universal Law of Generalization, defined over representations of arbitrary form.

Two questions arise regarding the scope of this derivation. The first question is whether the derivation is too general. Does the Universal Law hold for data that are not best modeled by data derived using a Euclidean metric? Evidence on this question appears to be sparse, perhaps because scaling techniques that embed items in Euclidean spaces are particularly well-developed and widely used. One piece of evidence that the law may extend to other metrics is given in Cunningham and Shepard (1974). Confusability data for Morse Code signals collected by Rothkopf (1957) were analysed by a very general scaling method, which makes only the metric assumptions. These data showed qualitatively the same pattern as in conventional non-metric multidimensional scaling analysis, consistent with the Universal Law. Moreover, Tenenbaum and colleagues (e.g., Tenenbaum, this issue) has used generalizations of multidimensional scaling, for example, modeling taxonomic hierarchies using tree structures, or causal dependencies in terms of directed causal graphs. These methods provide a rich set of tools for studying the scope of the Universal Law for broader classes of representation. 
The second question concerns whether the present analysis can be adapted to deal with the case of deliberate generalization, rather than mere stimulus confusion, as considered here---and, indeed, an interesting question concerns the strength of the empirical basis for the universal law in this case (see Chater \& Vitányi, 2003, for discussion). In any case, though, the analysis for confusability will turn out to supply a robust empirical generalization; and, as we now see, one that can be combined with assumptions about scale-invariance to provide an account in a different area of psychological theory: memory for serial order.

\section{Constructing models from principles: \\ The SIMPLE model of memory for serial order}

We noted at the outset that one of the attractions of general cognitive principles is that they provide a set of building blocks out of which models of specific cognitive phenomena may be constructed. Here, we provide a concrete example---indicating how a theory of memory for serial order, SIMPLE can be constructed by assuming both scaleinvariance and the simplicity-based generalization of Shepard's universal law (Brown, Neath \& Chater, in press; Surprenant, Neath \& Brown, 2006). SIMPLE was originally developed from ideas of scale-invariance, and strongly influenced by Shepard's Universal Law; the precise derivation presented here, relating simplicity and scaleinvariance together, is, however, new. An interesting future direction will be to see if it is possible to successfully constrain new theories using general cognitive principles; here, though, we consider the derivation of existing theories.

Let us begin, by simplicity, considering to-be-recalled items as indexed along a single psychological dimension: the length of time that has elapsed since they were encoded. Consider an experiment in which participants are presented with a sequence of, say, five items. They are then probed with a particular location in the sequence (i.e., asked "what was the fourth item in the sequence?"). Participants will give a range of responses, typically peaked, of course, around the correct answer (see Figure 6). Let us assume that the items themselves are easily distinguishable, and hence that errors arise in confusion between the times at which those items are encoded. We assume that times are 
encoded independently for each item and that errors occur because of uncertainty about these times.

This set-up can be viewed as an identification paradigm---in which the objects to be identified are locations in time, and their labels are the memory items, $I_{1}, I_{2} \ldots I_{5}$ (as in Figure 6). Thus, we can immediately apply a slightly variant of our generalization of Shepard's universal law, to describe the probability of confusion between items. Using the law in a simplified form (which follows directly from the derivation outlined above):

$$
\operatorname{Pr}\left(" I_{i} " \mid I_{j}\right) \propto e^{-a D\left(I_{i} \mid I_{j}\right)}
$$

Where $\operatorname{Pr}\left(" I_{i} " \mid I_{j}\right)$ is the probability of mistakenly responding with item $I_{i}$, when the correct item in that position is item $I_{j}$. Now, by the assumption that the confusion between item arises because of confusion between their times (rather than the items themselves), the key question is how to measure the code length $D\left(t_{i} \mid t_{j}\right)$ where $t_{i}$ and $t_{j}$ represent how long ago items $I_{i}, I_{j}$ occurred at the point of testing. ${ }^{\mathrm{xv}}$ Notice that his assumption crucially depends on viewing time as an explicitly encoded psychological dimension---otherwise the question of encoding time would not arise. Thus, we face the question: how complex is it to code time $t_{i}$, given the 'hint' of time $t_{j}$ ? By standard information theory, we can view this problem of coding in terms of probability---i.e., how likely is an item at time $t_{i}$ to occur, given that we know that an item at time $t_{j}$ has occurred. Specifically, we can use the relation:

$$
D\left(t_{i} \mid t_{j}\right)=-\log _{2} \operatorname{Pr}\left(t_{i} \mid t_{j}\right) \Delta t_{i}
$$

where $\Delta t_{i}$ is the precision of the encoding of $t_{j}$. Weber's Law (and scale-invariance) imply that $\Delta t_{i} \propto t_{i}$.

Scale invariance implies that the probability of encountering an item at $t_{i}$ (within a precision $\Delta t_{i}$ ), given a prior input $t_{j}$, must be the same as the probability of encountering $\alpha t_{i}$ (within a precision $\alpha \Delta t_{i}$ ), given a prior input $\alpha t_{j}$. That is, $\operatorname{Pr}\left(t_{i} \mid t_{j}\right) \Delta t_{i}$ is 
purely a function of the ratio between $t_{i}$ and $t_{j}$. We assume, further, that this probability is symmetrical, so that only the absolute value of the ratio is important. Let us define the function $r(x, y)$ to be $\min (x / y, y / x)$.

$$
\operatorname{Pr}\left(t_{i} \mid t_{j}\right) \Delta t_{i}=f\left(r\left(t_{i}, t_{j}\right)\right)
$$

A further application of scale-invariance is to assume the function $f$ itself is scaleinvariant, and hence a power function:

$$
f\left(r\left(t_{i}, t_{j}\right)\right) \propto\left(r\left(t_{i}, t_{j}\right)\right)^{b}
$$

Connecting (16-19) together, and simplifying, we can reason that:

$$
\begin{aligned}
& \operatorname{Pr}\left(" I_{i} " \mid I_{j}\right) \propto e^{-a D\left(I_{i} \mid I_{j}\right)}=e^{\left.a \log _{2} \operatorname{Pr}\left(t_{i} \mid t_{j}\right) \Delta t_{i}\right)} \\
& \left.\propto \operatorname{R}\left(t_{i} \mid t_{j}\right) \Delta t_{i}\right){ }^{c}=\boldsymbol{f}\left(r\left(t_{i}, t_{j}\right)\right) \stackrel{c}{\boldsymbol{j}}=\left(r\left(t_{i}, t_{j}\right)\right)^{d}
\end{aligned}
$$

where $a, b, c, d$ are arbitrary real constants. Hence, we can conclude that:

$$
\operatorname{Pr}\left(" I_{i} " \mid I_{j}\right) \propto\left(r\left(t_{i}, t_{j}\right)\right)^{d}
$$

Given an input $I_{j}$, we will produce some output, so that $\sum_{k} \operatorname{Pr}\left(" I_{k} " \mid I_{j}\right)=1$. Hence, we can normalize (21) to write the probability of responding $I_{i}$, when probed with the location of $I_{j}$, as:

$$
\operatorname{Pr}\left(" I_{i} " \mid I_{j}\right)=\frac{\left(r\left(t_{i}, t_{j}\right)\right)^{d}}{\sum_{k}\left(r\left(t_{k}, t_{j}\right)\right)^{d}}
$$

This equation is the core of a model of memory for serial order, SIMPLE (Brown, Neath $\&$ Chater, in press). The scope of SIMPLE is broad---indeed, much broader than the 
derivation here would suggest. Indeed, SIMPLE provides a fairly comprehensive model of data on serial and free recall, and provides a single mechanism that explains many data that are typically viewed as arising from a variety of distinct memory stores.

Figure 6 illustrates how SIMPLE provides excellent fits to data from a serial order reconstruction experiment (Nairne, 1992). Participants viewed lists of five items and rated them for pleasantness; at test participants were provided with the items required to arrange them in the order of presentation. The positional uncertainty gradients in Figure 6 show the different output positions into which an item was placed after $30 \mathrm{~s}$ (panel a); $4 \mathrm{~h}$ (panel b), and $24 \mathrm{~h}$ (panel c).

The model fit illustrated is with the model described in Equation 22, with the single parameter $d$ allowed to vary with retention interval (see Brown et al., in press, for details). We note that such scaling will be needed if performance is to be invariant with respect to the psychological spacing of items in temporal memory, as is (within limits) observed for the analogous case of absolute identification. In practice, perhaps due to the infeasibility of capturing all possible sources of memory interference, perfect scaleinvariance is seldom observed in estimated model parameters. This is not surprising, because changes of scale tend to lead to other changes (e.g., changes in the number of intervening items that may cause proactive or retroactive interference); and aspects of memory performance do have a characteristic scale (e.g., the rate at which items are rehearsed, or the rate at which they are reported at recall). Nonetheless, we suggest that, other things being equal, the more it is possible to minimize the effect of such factors (e.g., by using high distinctive items, with which intervening material does not interfere), the more closely observed behavior should fit with the predictions of scale invariance.

We have illustrated how general principles can be used as a basis for constructing cognitive models in specific domains. We do not, of course, take this process to be merely algorithmic---i.e., there will be many assumptions and idealizations that may be adopted, that will utilize basic principles in different ways (just as in the physical sciences). Nonetheless, working with a set of basic principles substantially constraints the process of developing specific theories; we may hope that these constraints will assist the development of cognitive models, rather than unhelpfully restricting it. The present derivation is an indication that, in some contexts at least, there is cause for optimism. 


\section{Extensions to identification and categorization}

The derivation that we have outlined can be applied more broadly. We noted above that SIMPLE depends on the confusability of pairs of items, and we focussed purely on a single dimension, time. But, if time is not different (at least, in relevant respects) from any other psychological dimension the same derivation can be applied to confusability between items on any dimension, e.g., weight, brightness, or loudness. Neath et al. (2006) note, indeed, that the application of SIMPLE successfully captures a wide range of phenomena in the task of absolute magnitude identification, where participants assign numerical labels to a fixed set of stimuli, with feedback (although see Stewart, Brown and Chater, 2005, for a more precise model).

If, by contrast, we consider more complex stimuli, distance is often not measured in physical terms (i.e., in terms of underlying physical parameters of sound pressure level, Newtons, and so on), but is left as a psychological primitive (and may, for example, be measured by the application of multi-dimensional scaling, Shepard, 1962a, 1962b). If we re-run the derivation above, but leave the distance $D$ unanalyzed, we obtain:

$$
\operatorname{Pr}\left(" I_{i} " \mid I_{j}\right)=\frac{e^{-D\left(t_{i}, t_{j}\right)}}{\sum_{k} e^{-D\left(t_{k}, t_{j}\right)}}
$$

which is a special case of the Nosofsky's (1986) model of identification (see also Luce, 1963; Shepard, 1957). Specifically, this special case lacks multiplicative "bias" weights, which can be used to capture response bias (i.e., an intrinsic tendency to choose some responses over others, independent of the stimulus). Note, too, that this formulation assumes, in line with Shepard's universal law, that generalization is an exponentially decreasing function of psychological distance. In some contexts, however, particularly when stimuli are readily confusable, a Gaussian, rather than exponential, function appears to provide a better fit with the empirical data (e.g., Nosofsky, 1986), i.e.,

$$
\operatorname{Pr}\left(" I_{i} " \mid I_{j}\right)=\frac{e^{-D\left(t_{i}, t_{j}\right)^{2}}}{\sum_{k} e^{-D\left(t_{k}, t_{j}\right)^{2}}}
$$


When the Gaussian model is appropriate, scale-invariance is violated - and hence, according to our earlier arguments, we may expect that this reveals some interesting cognitive discontinuity. Shepard (1986) and Ennis (1988) provide arguments supported this viewpoint - arguing that generalization follows the exponential universal law (following scale-invariance), except where the stimuli are difficult to discriminate, and hence where the accuracy of the representation of the stimuli is the limiting factor on performance. Thus, perceptual noise introduces a 'scale' when working at the limits of stimulus discriminability, just as motor noise introduces a scale, at very high frequencies in timing behavior (Gilden, Thornton \& Mallon, 1995), as we discussed above. In both cases, scale-invariant behavior informatively breaks down at the performance limits of the system.

Nosofsky (1986) notes a natural extension of this model to categorization based on labelled examples. Specifically, if the identifying labels for the examples are not unique, but can be shared between exemplars (i.e., examples with the same label are viewed as belonging to the same category), then the probability of producing a particular category label, $C$, is the sum of the probabilities of the exemplars, $i$, with that label (the $i$ such that $i \in C$. Specifically, this yields:

$$
\operatorname{Pr}\left(" I_{C} " \mid I_{j}\right)=\frac{\sum_{i \in C} e^{-D\left(t_{i}, t_{j}\right)}}{\sum_{k} e^{-D\left(t_{k}, t_{j}\right)}}
$$

This is Nosofsky's (1986) influential model of categorization, but, again, without free parameters to deal with the possibility of response bias.

\section{General Discussion}

\section{The scope of universal laws}

We noted at the outset that cognitive science looks to computer science, and allied disciplines, as its source of theoretical hypotheses---and that computer science, unlike physics, is not replete with universal laws. Indeed, it might be tempting to conclude that understanding the detailed computational principles of the brain might be no more 
amenable to general laws than the computational principles underpinning a complex piece of software, such as a word processor, statistical software package, a data-base, or, indeed, a model in traditional symbolic artificial intelligence. Such a piece of software is, of course, highly structured; the representations and methodologies it uses are carefully constrained; and there are many common features across different aspects of its behavior. But its behavior does not seem to exhibit quantitative universal laws.

It seems entirely possible, and indeed seems highly likely, that there are many aspects of cognition that must be understood in terms of specific representations and algorithms, which will not be neatly described by universal principles. But each individual case should, we suggest, be considered on its merits---and the possibility that general principles may combined to explain apparently complex phenomena should not be discounted. After all, the physical world too, is full of extremely diverse and idiosyncratic objects and processes (e.g., stars, comets, tides, earthquakes)---yet many aspects of these can usefully be understood in terms of basic physical theory.

Language provides a particularly interesting example. Chomsky (e.g., 1965, 1980) has influentially argued for a genetically encoded "universal grammar" which specifies information that is specific to language, and does not derive from functional considerations. If this picture is correct, then universal grammar is the antithesis of universal principles as stated here: it focuses, not on general cognitive principles, but on information specific to a particular (linguistic) domain. For example, binding constraints (Chomsky, 1981) provide elaborate, subtle and apparently arbitrary restrictions on coreference. For example, consider examples (26a-26d), where the subscripts indicate coreference, and asterisks indicate ungrammaticality.

$\mathrm{John}_{\mathrm{i}}$ likes himself $_{\mathrm{i}}$

*John ${ }_{\mathrm{i}}$ likes him $_{\mathrm{i}}$

$\mathrm{John}_{\mathrm{i}}$ said he $\mathrm{i}_{\mathrm{i} / \mathrm{j}}$ is happy

* $\mathrm{He}_{\mathrm{i}}$ said $\mathrm{John}_{\mathrm{i}}$ is happy

In (26a), the pronoun himself must refer to John; in (26b) it cannot. In (26c), the pronoun he may refer to John or to another person; in (26d), it cannot refer to John. While 
apparently arbitrary, these constraints can, however, be explained in terms of pragmatic factors (Levinson, 1987)---e.g., in (26b), the availability of the more specific himself to corefer with John has the implicature that if the less specific him is used, then coreference is not intended; in (26d), the use of John is unnecessarily specific, as a second pronoun would successfully co-refer to the first $h e$ ); and alternative explanations have been govern in terms of processing preferences (O'Grady, 2005; for a more general account of grammatical patterns as arising from processing constraints, see Hawkins, 1990); and both pragmatic and processing biases may themselves become part of the grammar over generations of language change, e.g., through grammaticalization (Hopper \& Traugott, 2003).

According to explanations of this type, it is possible that the linguistic patterns may arise from the confluence of a range of more basic cognitive principles, rather than being determined by genetically encoded language-specific principles. This raises the possibility that the interaction of relatively simple cognitive principles, of learning, processing, and pragmatics, might explain language structure, just as the interaction of basic physical principles is assumed to underpin the enormous variety and complexity of macroscopic physical phenomena. This style of explanation is consonant with much recent linguistic work, including construction grammar (e.g., Goldberg, 2006), and usagebased models of language more generally (e.g., Barlow \& Kemmer, 2000; Tomasello, 2003). Interestingly, it may also be consistent with Chomsky’s recent thinking. Hauser, Chomsky and Fitch (2002) suggest that much of language may be explicable by general cognitive mechanisms; and even the exception they suggest, recursion, appears to be widespread across cognitive domains (e.g., in planning and motor control) as well as arising across species (Conway \& Christiansen, 2001).

How far it proves to be possible to provide useful explanatory purchase on complex areas of cognition, including language, using an inventory of general cognitive principles is currently uncertain. Yet we suggest that the apparent variety and complexity of cognitive domains and phenomena should not be taken as evidence against general principles, any more than this is appropriate in the physical sciences. Indeed, this very variety should, we suggest, be a stimulus to the search for underlying generality. If 
general principles cannot be found, the cognitive sciences are likely to be highly fragmented, piecemeal, and intractable.

\section{Universal laws as reflections of the world}

If we suppose that there are broad regularities in mental phenomena, what is their origin? One possible source of universality is common processing mechanisms, whether understood and an algorithmic or neural level. For example, common principles in memory might arise from a common neural substrate, in terms of many or all memories might be encoded. Alternatively, however, it might be that some universal aspects of cognition have a functional, rather than a mechanistic, basis - they may arise as adaptations to the structure of the environment, and the agent's role within it. In an important sequence of papers, Anderson and colleagues have argued that many features of memory can be understood as adaptive in this way (Anderson \& Milson, 1989; Anderson \& Schooler, 2000; Schooler \& Anderson, 1997). The core idea is that, in the environment, items occurs in a highly clustered way. For example, if a particular word or object has occurred at one time, the probability that it will occur at later times is not constant, but decreases systematically with time. Specifically, this decrease with time is modelled be a power law---this is, of course, a further example of scale invariance. Anderson and colleagues make the reasonable assumption that an adaptive memory system should make items available in proportion to their likely occurrence. This leads to the prediction that forgetting should also have a power law, and thus scale-invariant, structure.

How can the adaptive and mechanistic viewpoints be distinguished? Perhaps the most straightforward line of argument concerns whether the regularity of interest arises across a range of different mechanistic systems---e.g., across a wide range of stimulus materials (presumably engaging different neural machinery), or across species. To the extent that very different underlying machinery leads to the same regularity, it is reasonable to suspect that the regularity has some adaptive basis. Thus, for example, temporal and spatial scale-invariance in the motor system seems to apply across a wide range of different effectors and tasks, ranging from handwriting, to gross body movements (e.g., Viviani \& Terzuolo, 1980). A second line of argument concerns 
whether the cognitive regularity follows 'bumps' in environmental structure. For example, human perception is roughly invariant to pitch (e.g., the same tune can readily be identified, even if shifted through several octaves); but speech perception is not invariant in this way. Thus, we have the familiar observation that music played at the wrong speed sounds only mildly odd until singing begins. From an adaptive point of view, this makes good sense---because the human vocal tract, and hence the sounds that it produces, have a particular scale, to which the auditory/speech processing system is attuned. Anderson and Schooler (1991) use this type of argument in supporting their rational analysis of memory retrieval based on the repetition structure in the environmental. Suppose that a particular item is presented ten times over a period of time; or in rapid succession. On the latter account, a natural assumption of an adaptive memory system adjusts its assessment of how likely an item is to recur, based on the spacing of occurrences in learning, and the spacing between the last such item, and the test item. Thus, if we fix the gap between the last study item and test to be, say, 20 trials, then memory should be better if previous items were spaced at roughly 20 trial intervals, rather than spaced much more densely. Thus, we should expect that forgetting should be slower, if the prior items are further back in time---a phenomenon that appears counterintuitive, in the light of the natural assumption that memory traces are active in proportion to their recency. Nonetheless, such 'spacing' effects are observed (e.g., Bahrick, 1979; Glenberg, 1976), providing evidence for an adaptive interpretation of the scaling properties of memory.

An important open question concerns which aspects of the scale-invariance in cognition should be viewed as reflections of scale-invariant environmental structure, and which should be viewed as arising from cognitive mechanisms. We suggest that this question should be addressed piecemeal for each scale-invariant aspect of cognition---i.e., we do not propose that there is a single 'unified' origin for scale-invariance in cognition. Scale-invariance is, after all, a null hypothesis, in the sense that it proposes the absence of effects that are specific to particular scales. Indeed, rather than attempting to provide a 'deep' explanation of this 'null' case, it may, in general, be more appropriate to focus attention on explaining departures from scale-invariance---what might term 'cognitive 
bumps'---and whether these appear to be best explained in mechanistic or functional terms. 


\section{References}

Adelson, E. H. (2000). Lightness Perception and Lightness Illusions. In M. S. Gazzaniga (Ed.) The New Cognitive Neurosciences ( $2^{\text {nd }}$ Edition) (pp 339-351), Cambridge, MA: MIT Press.

Anderson, J. R. (1983). The architecture of cognition. Cambridge, MA: Harvard University Press.

Anderson J. R., \& Milson R. (1989). Human memory: An adaptive perspective. Psychological Review, 96, 703-719.

Anderson, J. R., \& Schooler, L. J. (1991). Reflections of the environment in memory. Psychological Science, 2, 396-408.

Anderson J. R., \& Schooler L. J. (2000). The adaptive nature of memory. In: Tulving E, Craik F. I. M. (eds.) Handbook of Memory (557-570). Oxford University Press, New York.

Attneave, F., \& Frost, R. (1969). The determination of perceived tridimensional orientation by minimum criteria. Perception and Psychophysics, 6, 391-396.

Bahrick, H.P. (1979). Maintenance of knowledge: Questions about memory we forgot to ask. Journal of Experimental Psychology: General, 108, 296-308.

Balsam, P. D., Fairhurst, S. \& Gallistel, C. R. (2006). Pavlovian contingencies and temporal information. Journal of Experimental Psychology: Animal Behavior Processes, 32, 284-294.

Barenblatt, G. I. (1996). Scaling, self-similarity, and intermediate asymptotics. Cambridge: Cambridge University Press.

Barlow, H. B. (2001). The exploitation of regularities in the environment by the brain. Behavioral and Brain Sciences, 24, 602-607.

Barlow, M., \& Kemmer, S. (2000). (eds), Usage-Based Models of Language. Stanford, CA: CSLI Press.

Bennett, C. H., Gács, P., Li, M., Vitányi, P., \& Zurek, W. (1998). Information Distance. IEEE Transactions on Information Theory, 44, 1407-1423. 
Bienenstock, E., Geman, S, \& Potter, D. (1998). Compositionality, MDL Priors, and Object Recognition. In M. C. Mozer, M. I. Jordan \& T. Petsche (Eds.), Advances in Neural Information Processing Systems 9. Cambridge, MA: MIT Press.

Bjork, R. A., \& Whitten, W. B. (1974). Recency-sensitive retrieval processes in longterm free recall. Cognitive Psychology, 6, 173-189.

Blakemore, C. (ed.) (1990) Vision: Coding and efficiency. Cambridge, England: Cambridge University Press.

Brent, M. R. \& Cartwright, T. A. (1996). Distributional regularity and phonotactic constraints are useful for segmentation. Cognition, 61, 93-126.

Brown, G. D. A., Neath, I., \& Chater, N. (in press). A temporal ratio model of memory. Psychological Review.

Bush, R.R., \& Mosteller, F. (1951). A model for stimulus generalization and. discrimination. Psychological Review, 58, 413-423.

Carlton, E., \& Shepard, R. N. (1990a). Psychologically simple motions as geodesic paths: I. Asymmetric objects. Journal of Mathematical Psychology, 34, 127-188.

Carlton, E. H., \& Shepard, R. N. (1990b). Psychologically simple motions as geodesic paths. II. Symmetric objects. Journal of Mathematical Psychology, 34, 189-228.

Chandler, D. M. \& Field, D. J. (2007). Estimates of the information content and dimensionality of natural scenes from proximity distributions. Journal of the Optical Society of America A, 24, 922-941.

Chater, N. (1996) Reconciling simplicity and likelihood principles in perceptual organization. Psychological Review, 103, 566-581

Chater, N. (1999). The search for simplicity: A fundamental cognitive principle? Quarterly Journal of Experimental Psychology, 52A, 273-302.

Chater, N. (2005). A minimum description length principle for perception. In M. Pitt, \& I. Myung (Eds.) Advances in minimum description length: Theory and applications. Cambridge, MA: MIT Press.

Chater, N., \& Brown, G. D. A. (1999). Scale invariance as a unifying psychological principle. Cognition, 69, B17-B24. 
Chater, N., Tenenbaum, J. B., \& Yuille, A. (2006). Probabilistic models of cognition: Conceptual foundations. Trends in Cognitive Sciences, 10, 292-293.

Chater, N., \& Vitányi, P. M. B. (2002). Simplicity: A unifying principle in cognitive science? Trends in Cognitive Sciences, 7, 19-22.

Chater, N., \& Vitányi, P. M. B. (2003). The generalized universal law of generalization. Journal of Mathematical Psychology, 47, 346-369.

Chater, N., \& Vitányi, P. M. B. (in press). 'Ideal learning' of natural language: Positive results about learning from positive evidence. Journal of Mathematical Psychology.

Chechile, R.A. (2006). Memory hazard functions: A vehicle for theory development and test. Psychological Review, 113, 31-56.

Chomsky, N. (1955). The Logical Structure of Linguistic Theory. Plenum Press, New York.

Chomsky, N. (1965). Aspects of the theory of syntax. Cambridge, MA: MIT Press.

Chomsky, N. (1980). Rules and representations. Behavioral and Brain Sciences, 3, 1-61

Chomsky, N. (1981). Lectures on Government and Binding. Dordrecht: Foris Publications.

Conway, C., \& Christiansen, M. (2001). Sequential learning in non-human primates. Trends in Cognitive Science, 5, 539-546.

Cunningham, J. N., \& Shepard, R. N. (1974). Monotone mapping of similarities into a general metric space. Journal of Mathematical Psychology, 11, 335-63.

Davelaar, E. J., Goshen-Gottstein, Y., Ashkenazi, A., Haarmann, H. J., \& Usher, M. (2005). The demise of short-term memory revisited: empirical and computational investigations of recency effects. Psychological Review, 112, 3-42.

Dawid, A. P. (1984). Present position and potential developments: some personal views. Statistical theory: The prequential approach (with Discussion). Journal of the Royal Statistical Society A, 147, 278-292.

Dowman, M. (2000) Addressing the Learnability of Verb Subcategorizations with Bayesian Inference. In Gleitman, L. R. \& Joshi, A. K. (Eds.) Proceedings of the Twenty-Second Annual Conference of the Cognitive Science Society. Mahwah, New Jersey: Erlbaum. 
Duncan, J. (1984). Selective attention and the organization of visual information. Journal of Experimental Psychology: General, 114, 501-517.

Ennis, D. M. (1988). Confusable and discriminable stimuli: Comment on Nosofsky (1986) and Shepard (1986). Journal of Experimental Psychology: General, 117, 408-4 11 .

Falk, R., \& Konold, C. (1997) Making sense of randomness: Implicit encoding as a bias for judgment. Psychological Review, 104, 301-318.

Farrell, J. E., \& Shepard R. N. (1981). Shape, orientation, and apparent rotational motion. Journal Experimental Psychology: Human Perception and Performance, 7, 477-86.

Feldman, J. (2000) Minimization of Boolean complexity in human concept learning. Nature, 407, 630-633.

Feldman, J. (2006) An algebra of human concept learning. Journal of Mathematical Psychology, 50, 339-368.

Field D. J. (1987). Relations Between the Statistics of Natural Images and the Response Profiles of Cortical Cells. Journal of the Optical Society of America A, 4, 23792394.

Fitts, P. M. (1954). The information capacity of the human motor system in controlling the amplitude of movement. Journal of Experimental Psychology, 47, 381-391.

Fodor, J. A. (1983). Modularity of mind. Cambridge, MA: MIT Press.

Fodor, J. D. and Crain, S. (1987). Simplicity and generality of rules in language acquisition. In B. MacWhinney (ed) Mechanisms of Language Acquisition (pp. 3563). Hillsdale, NJ: Lawrence Erlbaum.

Fodor, J. A., \& Pylyshyn, Z. (1988). Connectionism and cognitive architecture. Cognition, 28, 3-72.

Gabaix, X. (1999). Zipf's Law and the Growth of Cities. American Economic Review, 89, 129-132.

Gallistel, C. R., \& Gibbon, J. (2000). Time, rate and conditioning. Psychological Review, $107,289-344$.

Garey, M. and D. Johnson, (1979). Computers and Intractability; A Guide to the Theory of NP-Completeness. New York: W. H. Freeman.

Garner, W. (1974) The Processing of Information and Structure. Potomac, MD: Erlbaum. 
Gilden, D. L., Thornton, T., \& Mallon, M. W. (1995). 1/f noise in human cognition. Science, 267, 1837-1839.

Gisiger, T. (2001), Scale invariance in biology: coincidence or footprint of a universal mechanism? Biological Reviews of the Cambridge Philosophical Society, 76, 161209.

Glenberg, A. M. (1976). Monotonic and nonmonotonic lag effects in paired-associate and recognition memory paradigms. Journal of Verbal Learning and Verbal Behavior, 15, 1-16.

Glenberg, A. M., Bradley, M. M., Kraus, T. A., \& Renzaglia, G. J. (1983). Studies of the long-term recency effect: Support for a contextually guided retrieval hypothesis. Journal of Experimental Psychology: Learning, Memory, and Cognition, 9, 231255.

Goldberg, A. (2006). Constructions at work. Oxford: Oxford University Press.

Goldberger, A. L., Amaral, A. N., Hausdorff, J. M., Ivanov, P., Peng, C.-K., \& Stanley, H. E. (2002). Fractal dynamics in physiology: Alterations with disease and aging. Proceedings of the National Academy of Science. 99(Suppl. 1), 2466-2472.

Goldsmith, J. (2001). Unsupervised learning of the morphology of a natural language. Computational Linguistics, 27, 153-198.

Griffiths, T.L., \& Tenenbaum, J.B. (2003) Probability, algorithmic complexity, and subjective randomness. Proceedings of the 25th Annual Conference of the Cognitive Science Society.

Grünwald, P., Myung, I. J. \& Pitt, M. (Eds) (2005). Advances in Minimum Description Length: Theory and Applications. Cambridge, MA: MIT Press.

Hahn, U., Chater, N., \& Richardson, L. B. C. (2003). Similarity as transformation. Cognition, 87, 1-32.

Handel, P. H., \& Chung, A. L. (Eds.). (1993). Noise in physical systems and 1/f fluctuations. New York: American Institute of Physics.

Hauser, M. D., Chomsky, N., \& Fitch, W. T. (2002). The Faculty of Language: What Is It, Who Has It, and How Did It Evolve? Science, 298, 1569-1579.

Hawkins, J.A. (1990). A parsing theory of word order universals. Linguistic Inquiry, 21, 223-261. 
Heathcote, A., Brown, S. \& Mewhort, D. J. (2000). The power law repealed: the case for an exponential law of practice. Psychonomic Bulletin \& Review, 7, 185-207.

Heit, E. (2000). Properties of inductive reasoning. Psychonomic Bulletin \& Review, 7 , $569-592$.

Hochberg, J. \& McAlister, E. (1953). A quantitative approach to figure "goodness." Journal of Experimental Psychology, 46, 361-364.

Hopper, P. J., \& Traugott, E. C. (2003). Grammaticalization ( $2^{\text {nd }}$ edition). Cambridge: Cambridge University Press.

Ijiri, Y., \& Simon, H. A. (1977). Skew Distributions and the Size of Business Firms. Amsterdam: North Holland.

Kelso, J. S. (2000). Dynamic Patterns: The Self-Organization of Brain and Behavior. Cambridge, MA: MIT Press.

Koffka, K. (1935). Principles of Gestalt psychology. London: Routledge and Kegan Paul. Leeuwenberg, E. L. J. (1971). A perceptual coding language for perceptual and auditory patterns. American Journal of Psychology, 84, 307-349.

Leeuwenberg, E. L. J., \& Boselie, F. (1988). Against the likelihood principle in visual form perception. Psychological Review, 95, 485-491.

Levinson, S. (1987). Pragmatics and the grammar of anaphora: A partial pragmatic reduction of binding and control phenomena. Journal of Linguistics, 23, 379-434.

Lewicki, M. S. (2002). Efficient coding of natural sounds. Nature Neuroscience, 5, 356363.

Li, M., \& Vitányi, P. (1997). An introduction to Kolmogorov complexity theory and its applications (2nd edition). Berlin: Springer.

Luce, R. D. (1963). Detection and recognition. In R. D. Luce, R. R. Bush, \& E. Galanter (Eds.), Handbook of mathematical psychology (pp. 103-189). New York: Wiley.

Mantegna, R. N., \& Stanley, H. E. (1995). Scaling behaviour in the dynamics of an economic index, Nature, 376, 46-49.

Marr, D. C., \& Poggio, T. (1976). Cooperative computation of stereo disparity. Science, 194, 283-287 
Maylor, E. A., Chater, N., \& Brown, G. D. A. (2001). Scale invariance in the retrieval of retrospective and prospective memories. Psychonomic Bulletin \& Review, 8, 162167.

Moore, B. C. J. (2003). An Introduction to the Psychology of Hearing (5th Ed), San Diego, CA: Academic Press.

Mumford, D. (1996). Pattern theory: a unifying perspective. In D. C. Knill and W. Richards, (Ed.) Perception as Bayesian Inference (pp. 25-62), Cambridge, UK: Cambridge University Press.

Nairne, J.S. (1992). The loss of positional certainty in long-term memory. Psychological Science, 3, 199-202.

Neath, I., \& Brown, G.D.A. (2006). Further applications of a local distinctiveness model of memory. Psychology of Learning and Motivation, 46, 201-243.

Neath, I., Brown, G.D.A., McCormack, T., Chater, N., \& Freeman, R. (2006). Distinctiveness models of memory and absolute identification: Evidence for local, not global, effects. Quarterly Journal of Experimental Psychology, 59, 121-135.

Newell, A. (1990). Unified Theories of Cognition. Cambridge, MA: Harvard University Press

Newell, A., \& Rosenbloom, P. S. (1981). Mechanisms of skill acquisition and the law of practice. In J. R. Anderson (Ed.), Cognitive skills and their acquisition. (pp. 1-51). Hillsdale, NJ: Lawrence Erlbaum Associates, Inc.

Norris, D., McQueen, J. M., \& Cutler, A. (2000). Merging information in speech recognition: Feedback is never necessary. Behavioral and Brain Sciences, 23, 299370.

Nosofsky, R. M. (1986), Attention, similarity, and the identification-categorization relationship. Journal of Experimental Psychology: General, 115, 39-57.

Oaksford, M., \& Chater, N. (1998). Rationality in an uncertain world. Hove, England: Psychology Press.

O’Grady, W. (2005). Syntactic carpentry: An emergentist approach to syntax. Mahwah, NJ: Erlbaum.

Pothos, E., \& Chater, N. (2002). A simplicity principle in unsupervised human categorization. Cognitive Science, 26, 303-343. 
Pylyshyn, Z. W. (Ed.) (1987). The robot's dilemma: The frame problem in artificial intelligence. Norwood, NJ: Ablex.

Richards, W. (Ed.) (1988). Natural Computation, Cambridge, MA: MIT Press.

Rissanen, J. (1987). Stochastic Complexity. Journal of the Royal Statistical Society, Series B, 49, 223-239.

Rothkopf, E. Z. (1957). A measure of stimulus similarity and errors in some pairedassociate learning tasks. Journal of Experimental Psychology, 53, 94-101.

Rumelhart, D. E., McClelland, J. L., \& the PDP Research Group (1986). Parallel Distributed Processing: Explorations in the Microstructure of Cognition, Volumes 1 and 2. Cambridge, MA: MIT Press.

Russell, S. J., \& Norvig, P. (2003). Artificial Intelligence: A Modern Approach, 2nd Edition. Upper Saddle River, NJ: Prentice-Hall.

Schooler, L. J. \& Anderson, J. R. (1997). The role of process in the rational analysis of memory. Cognitive Psychology, 32, 219-250.

Shanks, D. R. (1995). The psychology of associative learning. Cambridge: Cambridge University Press.

Schmidt, R.A., Zelaznik, H., Hawkins, B., Frank, J. S., \& Quinn, J. T. J. (1979). Motoroutput variability: a theory for the accuracy of rapid motor acts. Psychological Review, 47, 415-451.

Shepard, R. N. (1957). Stimulus and response generalization: a stochastic model relating generalization to distance in psychological space. Psychometrika, 22, 325-345.

Shepard, R. N. (1958a). Stimulus and response generalization: deduction of the generalization gradient from a trace model. Psychological Review, 65, 242-56.

Shepard, R. N. (1958b). Stimulus and response generalization: tests of a model relating generalization to distance in psychological space. Journal of Experimental Psychology, 55, 509-23.

Shepard, R. N. (1962a). The analysis of proximities: Multidimensional scaling with an unknown distance function. I. Psychometrika, 27, 125-40.

Shepard, R. N. (1962b). The analysis of proximities: Multidimensional scaling with an unknown distance function. II. Psychometrika, 27, 219-46. 
Shepard, R. N. (1964). Attention and the metric structure of the stimulus space. Journal of Mathematical Psychology, 1, 54-87.

Shepard, R. N. (1965). Approximation to uniform gradients of generalization by monotone transformations of scale. In D. Mostofsky (Ed.) Stimulus generalization. (pp. 94-110). Palo Alto, CA.

Shepard, R. N. (1966). Metric structures in ordinal data. Journal of Mathematical Psychology, 3, 287-315.

Shepard, R. N. (1980). Multidimensional scaling, tree-fitting, and clustering. Science, $210,390-398$.

Shepard, R. N. (1982). Geometrical approximations to the structure of musical pitch. Psychological Review, 89, 305-333.

Shepard, R. N. (1986). Discrimination and generalization in identification and classification: Comment on Nosofsky. Journal of Experimental Bsychology: General, 115, 58-61.

Shepard, R. N. (1987). Toward a universal law of generalization for psychological science. Science, 237, 1317-1323.

Shepard, R. N. (1994). Perceptual-cognitive universals as reflections of the world. Psychonomic Bulletin \& Review, 1, 2-28.

Shepard R. N., \& Cooper, L. A. (1982). Mental images and their transformations. Cambridge, MA: MIT Press.

Shepard R. N., \& Zare S.L. (1983). Path-guided apparent motion. Science, 220, 632-634.

Smolensky, P. \& Legendre, G. (2006). The harmonic mind: From neural computation to optimality-theoretic grammar Vol. 1: Cognitive architecture; vol. 2: Linguistic and Philosophical Implications. Cambridge, MA: MIT Press.

Soechting, J. F., \& Lacquaniti, F. (1981). Invariant characteristics of a pointing in man. Journal of Neuroscience, 1, 710-720.

Stevens, S. S. (1957). On the psychophysical law. Psychological Review, 64, 153-181.

Stewart, N., Brown, G.D.A., \& Chater, N. (2005). Absolute identification by relative judgment. Psychological Review, 112, 881-911. 
Surprenant, A. M., Neath, I., \& Brown, G. D. A. (2006). Modeling age-related differences in immediate memory using SIMPLE. Journal of Memory and Language, 55, 572-586.

Tenenbaum, J. B. (1999). Bayesian modeling of human concept learning. In M.. Kearns, S. Solla \& D. Cohn (Eds). Advances in Neural Information Processing Systems 11 (pp. 59-65). Cambridge, MIT Press.

Tenenbaum, J. B. (this issue). Cognitive Science.

Tenenbaum, J. B., \& Griffiths, T. L. (2001). Generalization, similarity, and Bayesian inference. Behavioral and Brain Sciences, 24, 629-641.

Tenenbaum, J.B., Griffiths, T. L., \& Niyogi, S. (2007). Intuitive theories as grammars for causal inference. In Gopnik, A., \& Schulz, L. (Eds.), Causal learning: Psychology, philosophy, and computation. Oxford University Press.

Tomasello, M. (2003). Constructing a language: A usage-based theory of language acquisition. Cambridge, MA: Harvard University Press.

Tversky, A. (1977). Features of similarity. Psychological Review, 84, 327-352.

Vitányi, P. M. B., \& Li, M. (2000). Minimum description length induction, Bayesianism and Kolmogorov complexity. IEEE Transactions on Information Theory, 46, 446464.

Viviani, P., \& Terzuolo, C. (1980). Space-time invariance in motor skills. InG. E. Stelmach \&, J. Requin (Eds.), Tutorials in Motor Behavior (pp. 525-533). NorthHolland, Amsterdam.

Wallace, C.S. \& Freeman, P.R. (1987). Estimation and Inference by Compact Coding, Proceedings of the Royal Statistical Society B, 49, 240-265.

Weber, E.H. (1996) De tactu. Annotationes anatomicae et physiologicae. Leipzig: Koehler. Translated in Ross, H.E. and Murray, D.J. (Eds) E.H. Weber on the tactile senses. 2nd edition. Hove, UK: Taylor \& Francis (Original work published 1834).

West, G. B. \& James H. Brown, J. H. (2005). The origin of allometric scaling laws in biology from genomes to ecosystems: towards a quantitative unifying theory of biological structure and organization. Journal of Experimental Biology, 208, 15751592. 
Wing, A. M. (1980). The long and short of timing in response sequences. In G. E.

Stelmach \& J. Requin (Eds.), Tutorials in motor behavior (pp.469-486).

Amsterdam: North Holland.

Wing, A. M. (2000). Motor control: Mechanisms of motor equivalence in handwriting. Current Biology, 10, R245-R248.

Wixted, J. T. \& Ebbesen, E. B. (1997). Genuine power curves in forgetting: A quantitative analysis of individual subject forgetting functions. Memory and Cognition, 25, 731-739.

Wolpert, D. M., \& Ghahramani, Z. (2004). Computational Motor Control. In M.S. Gazzaniga (Ed.), The Cognitive Neurosciences III (pp. 485-494), Cambridge, MA: MIT Press

\section{Figure titles and captions}

Figure 1. Self-similarity of power laws.

Changes of scale on both $x$ and $y$ axes simultaneously leave the function invariant. Thus, the form of the function cannot provide information about absolute scale. Power laws exhibit scale invariance.

Figure 2. Scale-invariance and Weber's Law.

If we assume that sensory magnitudes (here, judgements of distance) have scale invariant sensory noise, then this implies that the ratio of magnitude to error will be constant. This is Weber's Law. At the limits of the sensory system (e.g., when magnitudes become extremely small), error will increase disproportionately. Thus, the departure from scaleinvariance reveals the mechanistic properties of the sensory system. By contrast, where scale-invariance holds, this provides little diagnostic information concerning cognitive mechanisms (see Chater \& Brown, 1999).

Figure 3. Scale-invariance in recall over different time intervals. Maylor, Chater and Brown (2001) asked people to recalls events that had occurred (a) or were going to occur (b) within a day, week or year. The number of items retrieved was 
invariant to the time interval probed. This implies that the 'salience' of items considered worth reporting for each of these intervals scales with the size of that interval. In the absence of such scaling of salience, it would be natural to assume that more items should be retrieved for longer intervals, because all items that occurred over a short interval necessarily also occurred during the longer interval. Data replotted from Maylor et al (2001).

Figure 4. Scale-invariance, the ratio rule, and serial position.

a. Three learning schedules, which differ purely by temporal scale. Thus the ratio between the intervals between items, and the interval from the final study item to test is held constant. The ratio rule (Bjork \& Whitten, 1974; Glenberg et al., 1983) states that the slope of the recency curve should depend only on this ratio---i.e., should be invariant across these cases, as is observed.

Figure 5. Simplicity and Gestalt principles.

The Gestalt principles of perceptual organization can be explained in terms of the simplicity principle. a. Grouping by similarity. We assume that objects are encoded separately; or, more modestly, that sharing information is more difficult between objects than it is within objects. This assumption is consistent with object-based views of attention (e.g., Duncan, 1984). If the top array is organized in terms of columns, colour is easy to encode - each column is either black or white. Hence, coding colour requires just 1 bit of information for each column. By contrast, if the array is organized by rows, each row has an irregular structure, which must be coded separately, and will require a longer code length. Hence, according to the simplicity principle, the column organization is preferred; more generally, groups containing similar items can be encoded more briefly. b. Common fate. Objects with the same motion, such as flocks of birds, tend to be grouped together. When the objects are grouped together, the stimulus can be coded by a vector indicating the location of each object, and a single vector for the movement of each group. The ungrouped code would require a separate vector for the motion of each item. 
Figure 6. Memory for serial order over three time-scales.

Proportion of responses in a serial order reconstruction task. Each graph indicates the serial position of the items that participants produce, typically peaking on the correct serial position. The three panels indicate performance for delays before testing of $30 \mathrm{~s}, 4 \mathrm{~h}$ and 24. Notice that, while performance is clearly degraded after a longer retention interval, the pattern of results is qualitatively the same. The solid lines give fits from SIMPLE are shown alongside each set of data. Data replotted from Nairne (1992).

i For example, Barenblatt (1996) describes the example of how Taylor (1950a, 1950b) was able to derive that the radius of the fireball of an atomic explosion is proportional to $E^{1 / 5} t^{2 / 5} \rho^{-1 / 5}$ where $E$ is the energy released, $t$ the time elapsed since detonation, and $\rho$ the initial air pressure, purely from considerations of scale-invariance. This analysis agreed astonishingly well with experimental data. ii This is an example of the so-called universal prior, that the prior probability of any object $x$ is $2^{-}$ ${ }^{K(x)}$. One justification of this prior is that it is, in a specific sense, as neutral as possible. That is, if any computable prior gives some $x$ a certain probability, the universal prior gives $x$ 'nearly as much' probability---i.e.., for any computable prior Pr, there is a constant $m$ such that for all $x, m 2^{-K(x)} \geq \operatorname{Pr}(x)$. For discussion, see Li and Vitányi (1997).

iii The class of possible models or hypotheses is restricted only by the requirement that the probability of the data must be computable (strictly, semi-computable from below, see Li \& Vitányi, 1997). This is a very mild restriction---and it seems reasonably to assume models with uncomputable predictions would be of limited practical utility to the cognitive system.

iv Intuitively, this result holds because $-\log _{2} \operatorname{Pr}(x)$ is the code length for $x$, given $\operatorname{Pr}$, according to standard information theory; and the Kolmogorov complexity, as the shortest code, must be at least this short. It is possible, but unlikely, that the converse is violated---i.e., that Pr can generate objects, $x$, which have much shorter description than the $-\log _{2} \operatorname{Pr}(x)$ from standard information theory. For example, suppose we consider a uniform distribution on strings of length $n$, so that for each $x, \operatorname{Pr}(x)=2^{-n}$. Standard information theory will assign each $\mathrm{x}$ a code length of $-\log _{2} \operatorname{Pr}(x)=\log _{2}\left(2^{-n}\right)=n$ bits of information. Some strings, e.g., $000 \ldots 000$ will have shorter codes (e.g., using a simple looping program); but there will be few of these, by a counting argument---i.e., while there are $2^{\mathrm{n}}$ strings of length $n$, there can only be at most $2^{k}$ codes of length $k$, where $k<n$, and hence at most a fraction $1 / 2^{n-k}$ strings of length $n$ which have codes as short as $k$. More generally, for any computable $\mathrm{Pr}$, a similar argument shows that the probability of generating an item with a shorter code is small. Thus, $K(x)$ approximates to $\operatorname{Pr}(x)$; and the argument extends to the conditional case, so that $\mathrm{K}(y \mid x)$ approximates $\operatorname{Pr}(y \mid x)$, up to a constant term, with high probability. 
$\mathrm{v} \quad$ This result suggests that induction is possible in principle, from the available data, given the very modest restriction that the data is generated by computable and/or probabilistic processes. A restriction, though, is that the simplicity principle cannot be implemented accurately in practice, because calculating Kolmogorov complexity is itself, in general, uncomputable. An interesting research question is far how the theoretical results we have outlined apply to practical approximations to the simplicity principle. The successful application of minimum description length, and related, methods in statistics and machine learning indicate that such approximations are often reliable in practice (Rissanen, 1987; Grünwald, Myung \& Pitt, 2005).

vi The divide between cases in which simplicity or probability is most usefully viewed as basic may, however, be less than straightforward. For example, Chandler and Field (2007) argue that, despite the intense study of the underlying physics and statistical structure of natural images, the best estimates of the information content of natural images comes from directly applying a coding method.

vii Kolmogorov complex is, notably, invariant up to a constant, between any two universal programming languages; and for these reason it is possible to develop an abstract theory of Kolmogorov complexity in general; and to prove asymptotic results such as the prediction theorem. Nonetheless, in deriving predictions concerning specific, finite, sets of sensory, linguistic, or other, data, the 'constant' that can be ignored in theoretical analysis, may turn out to be important. Thus, it may be important to have some prior knowledge of the representations in terms of which the cognitive system operates. Theories of representation are, though, highly developed in some areas of cognitive science; for example, linguistic theory provides a potential starting point for a theory of representations of linguistic knowledge. viii Interestingly, scale-invariance can also be viewed as providing an ignorance prior (albeit an improper prior-i.e., a prior probability that sums to greater than 1), over continuous quantities.

Kolmogorov complexity, by contrast, is most readily defined over discrete objects. Whether scaleinvariance may usefully be viewed as derivable from simplicity, or whether should be viewed as separate, is an interesting question for future research.

ix There are various variants of information distance, of which the formally most elegant is $\left.D_{\max }(x, y)=\max \{K(y \mid x), K(x \mid y)\}\right)$.

$\mathrm{x} \quad$ We say that a distance is normalized if, for every binary string $x, \sum_{y: y \neq x} 2^{-D(x, y)}<1$ where $y$ ranges over all other binary strings. If the constraint is violated by some cognitive metric, the constraint requires simply that all distances are scaled-up, e.g., by a multiplicative factor, until the constraint does hold. An admissible distance $D(x, y)$ is a total nonnegative function on the pairs $x ; y$ of binary strings that is 0 if and only if $x=y$; is symmetric, satisfies the triangle inequality, is upper-semicomputable (roughly, can be approximated from above arbitrarily closely, by a computational process) is normalized, and is a metric (in the standard mathematical sense).

xi Note that this derivation assumes that cognitive distance is well-approximated by the average conditional Kolmogorov complexity required to transform each item into the other (in line with the 
Representational Distorition theory of similarity, Hahn, Chater \& Richardson, 2003). Thus if, in some context, cognitive distance, e.g., of two locations on a computer screen, were closely related to physical distance, then the derivation would not hold---and, indeed, we should not predict Shepard's Universal Law to hold good.

${ }^{x i i}$ Note that this is a fairly innocuous assumption: we simply assume that the participant knows the relationship, in principle, between the inputs and the outputs. That is, if the confusion were between phonemes, we begin by assuming that participants already know the relevant inventory of phonemes, and hence can provide the appropriate response in the task, under ideal conditions. Errors in the task are presumed to arise when performance is difficult (e.g., under noise).

xiii This case does not arise in the identification paradigm. It does, however, arise in paired associate learning. Here, confusion errors are a function both of the similarity of the 'stimulus' items and the 'response' items, and also, potentially, similarity of the relationship between them. For example, if many of the pairs were synonyms and antonyms, this might substantially increase the probability of saying white in response to black. This type of case can, nonetheless, be analyzed given the machinery described here. xiv It might seem that the constant $B$ is not arbitrary, but has a fixed value, but in reality it is a free parameter, because Kolmogorov complexities themselves can be rescaled by a linear multiplicative factor. This corresponds to changing the number of primitive symbols in the assumed coding language. xv We assume that there is no absolute 'clock,' but that time in the past is estimated by reference to the present. 